\title{
Surface nanocavitation of titanium modulates macrophage activity
}

This article was published in the following Dove Press journal: International Journal of Nanomedicine

\author{
Marianne B Ariganellol,* \\ Dainelys Guadarrama \\ Bello 1,* \\ Alejandra Rodriguez- \\ Contreras' \\ Shayan Sadeghi' \\ Gaetano Isola' \\ Fabio Variola ${ }^{2,3}$ \\ Antonio $\mathrm{Nanci}^{1,4}$ \\ 'Laboratory for the Study of \\ Calcified Tissues and Biomaterials, \\ Department of Stomatology, Faculty \\ of Dental Medicine, Université de \\ Montréal, Montreal, QC, Canada; \\ ${ }^{2}$ Department of Mechanical \\ Engineering, University of Ottawa, \\ Ottawa, ON, Canada; ${ }^{3}$ Department \\ of Cellular and Molecular Medicine, \\ University of Ottawa, Ottawa, ON, \\ Canada; ${ }^{4}$ Department of Biochemistry \\ and Molecular Medicine, Faculty of \\ Medicine, Université de Montréal, \\ Montreal, QC, Canada \\ *These authors contributed equally \\ to this work
}

Correspondence: Antonio Nanci Faculté de Médecine Dentaire, Université de Montréal, CP 6128, Succursale Centre-Ville, Montreal, QC H3C 3J7, Canada

$\mathrm{Tel}+\mathrm{I} 5 \mathrm{I} 43435846$

Fax + I 5 I43432233

Email antonio.nanci@umontreal.ca
Background: Nanoscale surface modifications are widely touted to improve the biocompatibility of medically relevant materials. Immune cells, such as macrophages, play a critical role in the initial healing events following implantation.

Methods: To understand the response of macrophages to nanotopography better, we exposed U937-derived macrophages to a distinctive mesoporous titanium surface (TiNano) produced by a process of simple chemical nanocavitation, and to mechanically polished titanium (TiPolished) and glass coverslip (Glass) surfaces as controls. Cell numbers and morphology were evaluated. Osteopontin expression and that of the proinflammatory SPARC protein and its stabilin 1 receptor were analyzed. Release of inflammation-associated cytokines and chemokines was also measured.

Results: Compared to the two control surfaces, there were fewer U937 cells on TiNano, and these exhibited a more rounded morphology with long filopodia. The cells showed areas of punctate actin distribution, indicating formation of podosomes. Of the three proteins examined, only osteopontin's immunofluorescence signal was clearly reduced. Irrespective of the substrate, the cytokine assay revealed important variations in expression levels of the multiple molecules analyzed and downregulation in a number of chemokines by the TiNano surface.

Conclusion: These results indicate that macrophages sense and respond to the physicochemical cueing generated by the nanocavitated surface, triggering cellular and molecular changes consistent with lesser inflammatory propensity. Given the previously reported beneficial outcome of this mesoporous surface on osteogenic activity, it could be presumed that modulation of the macrophagic response it elicits may also contribute to initial bone-integration events.

Keywords: nanotopography, inflammation, osteopontin, SPARC, stabilin 1, cytokines

\section{Introduction}

Surgical implantation of biomaterials initiates a cascade of cellular and biochemical events that ultimately determine the quality of their integration in the body. Macrophages modulate immunoresponse during inflammation and healing, and thereby represent a key player in these events. The functional behavior of macrophages is dictated not only by the cytokine and cellular environment that is generated at a wound site but also by the biomaterial itself. Following implantation, one of the initial events is the adhesion of macrophages on the implant surface, a process that is highly dependent on its physicochemical properties. ${ }^{1,2}$ While there is general agreement that surface features have a determining influence on the inflammatory response, there are still some discrepancies regarding size. ${ }^{3}$ Because cell-matrix-substrate interactions occur on the nanoscale, surface topography at this scale can directly influence the cellular response. ${ }^{4}$ Based on these considerations, researchers have focused their efforts on modifying nanoscale surface topography with the objective of reducing detrimental inflammatory events that could lead to rejection or fibrous encapsulation. ${ }^{5}$ submit your manuscript Dovepress f 1 in

in 2 
Nanotopography-mediated macrophage activity strongly depends on the morphological characteristics of surface features. ${ }^{3,6}$ For instance, $\mathrm{Ma}$ et $\mathrm{al}^{7}$ found that $\mathrm{TiO}_{2}$ nanotubes with a diameter of $30 \mathrm{~nm}$ produced less inflammation compared to $80 \mathrm{~nm}$. On the other hand, Neacsu et $\mathrm{al}^{8}$ showed that similar surfaces with a mean diameter of $\sim 78 \mathrm{~nm}$ attenuated the inflammatory activity of RAW264.7 macrophages. Therefore, outcomes cannot simply be predicted based only on the size of surface features, but rather any relevant nanostructured topography must undergo an evaluation to obtain a reliable indication of inflammatory potential. Our group has been exploiting simple oxidative treatments to generate mesoporous $(<50 \mathrm{~nm})$ topographies on various metallic surfaces. ${ }^{9,10}$ The process, termed "nanocavitation" (formation of nanoscale spaces), ${ }^{11}$ has been used to modify the surfaces of biocompatible metals, such as titanium and Ti6Al4V alloy, CrCoMo, tantalum, ${ }^{9}$ and recently $316 \mathrm{~L} / 304$ stainless steel. ${ }^{11}$ In the case of titanium, the resulting nanocavitated surface consists of an intricate network of pits with diameters of 20-22 nm and depths of 10-20 nm. ${ }^{10}$ This mesoporous surface reliably and selectively influences various cell types and promotes osteogenic activity both in vitro ${ }^{9,12-14}$ and in vivo. ${ }^{15}$ Nanocavitated surfaces also simultaneously limit bacterial adhesion and growth. ${ }^{11,16,17}$ However, their inflammatory propensity remains to be defined.

The present work aims at probing the impact of nanocavitated titanium surfaces on mediating the initial inflammatory response. To this end, we have compared the response of U937-derived macrophages plated on titanium with a mesoporous surface (TiNano), polished titanium (TiPolished), and glass coverslips (Glass). Cell number and morphology were evaluated. Expression of osteopontin (OPN) and of the proinflammatory SPARC protein and its stabilin 1 receptor were analyzed by immunofluorescence. Release of inflammation-associated cytokines and chemokines was also measured. Altogether, our results demonstrated that the mesoporous surface network created favorably controlled the activity of macrophages in a way that was consistent with reduced inflammatory response. They also broaden our general understanding of how this important immune cell responds to biocompatible, yet foreign materials.

\section{Methods}

\section{Chemical nanocavitation of titanium samples}

Commercial grade 2 titanium disks $13 \mathrm{~mm}$ in diameter and $2 \mathrm{~mm}$ thick were polished, sonicated in distilled water, and rinsed with toluene (TiPolished). ${ }^{12}$ Nanocavitated titanium
(TiNano) samples were generated by immersing polished disks for 2 hours in a solution composed of equal volumes of $96 \% \mathrm{H}_{2} \mathrm{SO}_{4}$ (JT Baker, Phillipsburg, NJ, USA) and 30\% aqueous $\mathrm{H}_{2} \mathrm{O}_{2}$ (Thermo Fisher Scientific, Waltham, MA, USA) as previously reported. ${ }^{18}$ The two components of the oxidizing solution were mixed in a beaker and placed in an ice bath to control the exothermic reaction until the temperature stabilized at $22^{\circ} \mathrm{C}$. The disks were then introduced into the mixture (total volume in the beaker: $10 \mathrm{~mL} /$ disk) and kept under continuous agitation with a magnetic stir bar. Treated samples were then rinsed with distilled water, sonicated for 15 minutes in distilled water, and air-dried. Prior to use, control and treated disks were sterilized by immersion in $70 \%$ ethanol, followed by three rinses in PBS containing no $\mathrm{Ca}^{2+} / \mathrm{Mg}^{2+}$ (Thermo Fisher Scientific).

\section{Cell culture}

Human immortalized U937 cells (CRL1593.2; American Type Culture Collection (ATCC), Manassas, VA, USA) were maintained in suspension as promonocytes in RPMI 1640 medium supplemented with $25 \mathrm{mM}$ glucose, $10 \mathrm{mM}$ HEPES, $18 \mathrm{mM}$ sodium bicarbonate, $1 \mathrm{mM}$ sodium pyruvate, and $10 \%$ FBS (Thermo Fisher Scientific) as previously described. ${ }^{19}$ They were then seeded onto Glass and TiPolished as controls and TiNano at a density of $0.5 \times 10^{6}$ cells/disk. One hundred $\mathrm{nM}$ phorbol 12-myristate 13 -acetate ${ }^{20}$ was added to achieve differentiation. Cell cultures were carried out in an incubator at $37^{\circ} \mathrm{C}$ with $5 \% \mathrm{CO}_{2}$. Based on the progression of cell cultures in pilot analyses, cells were processed after 72 hours for scanning electron microscopy (SEM) and immunofluorescence.

\section{SEM}

Cells were fixed for 2 hours at room temperature (RT) in $0.1 \mathrm{M}$ sodium phosphate buffer ( $\mathrm{PB} ; \mathrm{pH}$ 7.2) containing $2.5 \%$ glutaraldehyde (EMS, Hatfield, PA, USA). Samples were postfixed with $1 \%$ aqueous osmium solution (1 hour at $4^{\circ} \mathrm{C}$ ) and washed three times ( 5 minutes) with PB. Then, cells were dehydrated in increasing aqueous ethanol concentrations $(30 \%-100 \%)$ and finally dried in a critical point dryer (CPD 030; Balzers, Hudson, NH, USA). Cells cultured on Glass were coated with a thin layer of carbon prior to observation to reduce surface charging of this substrate. Imaging was carried out with field-emission SEM (7400F; JEOL, Tokyo, Japan) using low voltages (1-1.5 kV).

\section{Immunofluorescence microscopy}

U937 cells cultured on each of the three substrates were first washed in $\mathrm{PB}$ and then fixed for 30 minutes at $4{ }^{\circ} \mathrm{C}$ in $4 \%$ 
freshly prepared paraformaldehyde solution in PB. After being washed three times in PB, cells were processed for immunofluorescence labeling at RT. Briefly, they were permeabilized with $0.5 \%$ Triton X-100 in PB for 10 minutes, followed by blocking with $5 \%$ skimmed milk, also in PB, for 1 hour. Cells were then incubated in a humidified environment for 2 hours with rabbit polyclonal antibodies to antihuman OPN at 1:500 dilution (LF123; courtesy of LW Fisher), bovine osteonectin (SPARC) at 1:400 (LF-BON1; courtesy of LW Fisher), and antihuman stabilin 1 at 1:200 (Millipore C-terminus AB6021). Corresponding goat secondary antibodies tagged with Alexa Fluor 488 (green fluorescence) or 594 (red fluorescence; Thermo Fisher Scientific) were used at a working dilution of 1:500. Omission of the primary antibody was used as control. All antibody dilutions were prepared in PB containing $0.5 \%$ milk protein. Rhodamine-phalloidin (red fluorescence, 1:150; Thermo Fisher Scientific) was used for actin labeling. Between each incubation step, samples were washed in PB (three times for 5 minutes). Titanium samples were placed cell side upon glass slides and covered with Glass using mounting medium containing DAPI to stain nuclei fluorescent blue (Thermo Fisher Scientific). Control cells grown on Glass were placed face down with DAPI mounting medium. Samples were then examined by epiluminescence under conventional, upright, and fluorescence microscopy (Axio Imager M2; Carl Zeiss, Jena, Germany) using the same exposure settings to estimate expression. For cell counts, a minimum of 1,700 cells were counted at $20 \times$ magnification.

\section{Phagocytosis of latex beads}

Phagocytic activity was measured as previously described. ${ }^{21}$ Briefly, U937 promonocytes were collected, plated onto Glass, TiPolished, and TiNano disks, and treated as described earlier for differentiation. They were then serum-starved for 2 hours (from 70 to 72 hours of incubation with only RPMI 1640 medium). Then, freshly sonicated ( 5 minutes) latex beads (L4655; Sigma-Aldrich Co., St Louis, MO, USA) were added $(6 \mu \mathrm{L} /$ well). After 3 hours, wells were rinsed with cold PBS to remove the excess of beads. Trypsin-EDTA $(0.25 \%$, $150 \mathrm{~mL}$ ) was added for 30 seconds and rinsed twice with PBS (5 minutes). Finally, cells were fixed in paraformaldehyde and stained for actin and nuclei as described earlier. To estimate macrophage phagocytic activity, a minimum of 100 cells were counted and expressed as a percentage of phagocytic cells.

\section{Cytokine release from macrophages}

To evaluate whether nanotopography elicited different inflammatory responses, the release of 40 cytokines and chemokines was quantified using a Bio-Plex 40 assay system at the Princess Margaret Genomics Center (Toronto, ON, Canada) according to the Bio-Rad human cytokine/ chemokine protocol. Briefly, U937 cells were cultured for 72 hours, $50 \mu \mathrm{L}$ culture medium was collected and incubated with Bio-Plex magnetic beads for 30 minutes at RT, and washed twice with $100 \mu \mathrm{L}$ wash buffer. Beads were subsequently washed again three times and incubated with $25 \mu \mathrm{L}$ detection antibody for 30 minutes. Streptavidin-phycoerythrin $(50 \mu \mathrm{L})$ were added to the beads for 10 minutes at RT. Beads were again washed and resuspended in $125 \mu \mathrm{L}$ assay buffer. Assays were read with a Luminex 100 reader and data analyzed using Bio-Plex Manager 6.0. Analyte concentrations are indicated in $\mathrm{pg} / \mathrm{mL}$.

\section{Statistical analyses}

Cell number, cell area, phagocytic activity, and cytokine release on the mesoporous surface were analyzed using oneway ANOVA at an overall significance level of 0.05 , and Bonferroni adjusted for multiple comparisons. All statistical analyses were carried out using OriginPro 8.5 software (OriginLab). Results are expressed as mean \pm SD. Values of $P<0.05$ were considered statistically different, while values above were not different. The results described are representative of three sets of experimental cultures, each carried out at different time points. For the cytokine-release study, results were obtained from a single experimental run with four samples for each substrate.

\section{Results and discussion}

Studies on biomaterial biocompatibility often focus on the response of tissue-specific cells (eg, osteoblasts for hardtissue implants, fibroblasts for soft-tissue implants). Such studies do not take into consideration the inflammatory response to biomaterials that occurs immediately after implantation. Given the macrophage proclivity for producing and releasing chemical mediators that can have direct (eg, enzymes) or indirect (signaling molecules) effects on the microenvironment surrounding the biomaterial, these cells have great capacity to direct downstream cellular events and affect the behavior of tissue-specific cells. Analysis of TNF $\alpha$, a proinflammatory cytokine, concluded that both macrophage morphology and their release of cytokines reflected the biocompatibility of a surface and correlated with each other. ${ }^{22}$

The present study investigated the response of macrophages to a modified titanium surface generated by a process of simple chemical nanocavitation. This surface was previously shown to have a manifest influence on 
fibroblasts, osteogenic, and stem cells. ${ }^{12,23,24}$ To characterize the macrophage response, our work distinctively analyzed a broad spectrum of parameters, including cell morphology and function and expression of proinflammatory molecules and cytokines.

\section{Characterization of titanium surfaces}

As previously reported, 9,10,13,25 treatment of titanium with a mixture of $\mathrm{H}_{2} \mathrm{SO}_{4} / \mathrm{H}_{2} \mathrm{O}_{2}$ predictably generates an amorphous $\mathrm{TiO}_{2}$ surface estimated to be $\sim 32-40 \mathrm{~nm}$ in thickness with a root-mean-square roughness of $\sim 17 \mathrm{~nm}$ determined by atomic-force microscopy. This surface layer comprises a network of nanopores (Figure 1) with diameters of 20-22 nm and depths of 10-20 nm. No distinctive topographical features were seen on TiPolished (Figure 1).

Surface chemical composition of the untreated sample surface indicated the presence of naturally formed titanium oxide with some contaminants, such as $\mathrm{C}, \mathrm{N}$, and $\mathrm{Si}^{10}{ }^{10}$ After chemical treatment, there were no contaminants except for $\mathrm{C}$, which is normally observed on oxide layers under atmospheric conditions. It is worth noting that no traces of $\mathrm{S}$ were observed, even though the samples were immersed in concentrated $\mathrm{H}_{2} \mathrm{SO}_{4} \cdot{ }^{10}$

\section{Cell morphology, growth, and spread}

Staining of nuclei with DAPI indicated that there were fewer U937 cells on TiNano compared to TiPolished, but no difference with Glass was found (Figure 2A). Quantitative analysis demonstrated that the difference was statistically significant (Figure 2B). This observation indicates cells adhered less on the mesoporous surface compared to the smooth one (Figure 2). Preliminary experiments with fully differentiated
U937 cells (as described earlier) yielded similar adhesion results (data not shown). However, unequivocally defining whether this difference was a result of differential adhesion only or a combination of adhesion and differentiation would require analyses at short time intervals after plating (eg, 12 hours).

Macrophages can adopt different migration modes defined by different cell morphologies, depending on the physical and chemical properties of specific environments. ${ }^{22,26,27}$ The amoeboid-migration mode is characterized by spherical cells with a small number of short protrusions. In the mesenchymal mode, cells display an elongated shape with multiple long protrusions. ${ }^{28}$ Field-emission SEM imaging (Figure 3) permitted the detailed study of cell morphology, revealing differences as a function of surface topography. As illustrated in the representative images shown in Figure $3 \mathrm{~A}$ and $\mathrm{B}$, macrophages spread less and adopted a more rounded profile on TiNano. They also occupied less surface area than cells on TiPolished (Figure 4).

The U937 macrophages on TiPolished showed cells with large cytoplasmic veils (red arrows in Figure 3A) surrounding the entire cell with several short filopodia emerging from them (Figure 3C). No such cytoplasmic veils were observed in cells on TiNano (Figure 3A), but these emitted numerous thin and long filopodial extensions (Figure 3C). Interestingly, cells differentiated on Glass presented a morphology that reflected aspects on both titanium surfaces (Figure 3).

Cells on the TiNano surface showed punctate actin expression (Figure 5C), which may be indicative of podosome formation (Figure 5C, enlarged areas). Podosomes are actin-rich structures involved in adhesion and invasion functions of macrophages. ${ }^{29}$ However, very little punctate
TiPolished

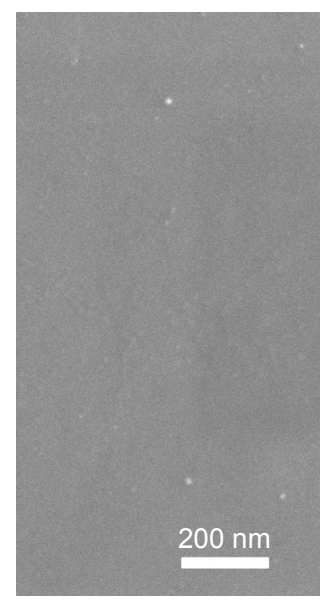

TiNano

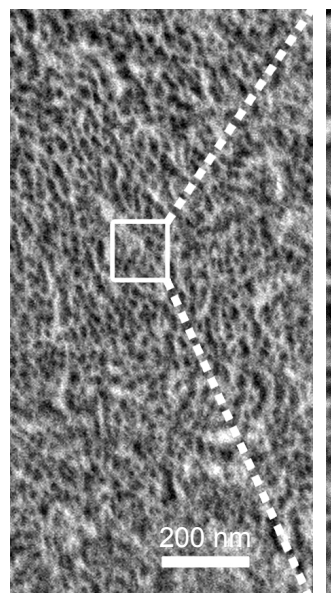

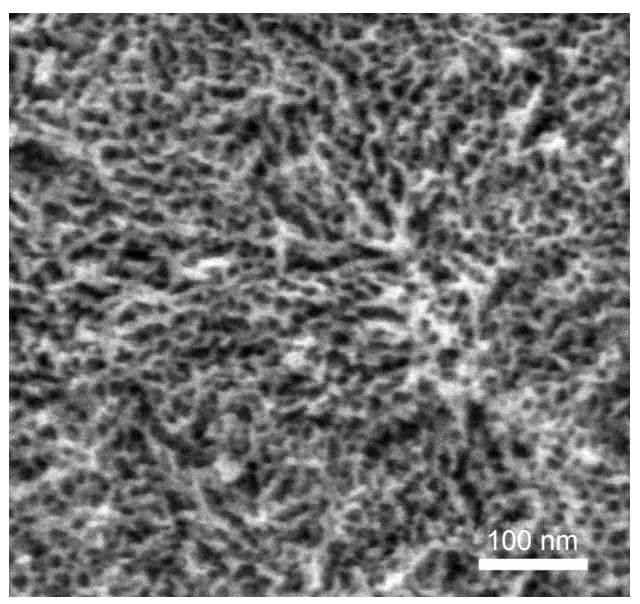

Figure I Field-emission scanning electron microscopy of untreated (TiPolished) and treated (TiNano) surfaces.

Abbreviations: TiPolished, polished titanium; TiNano, nanocavitated titanium. 

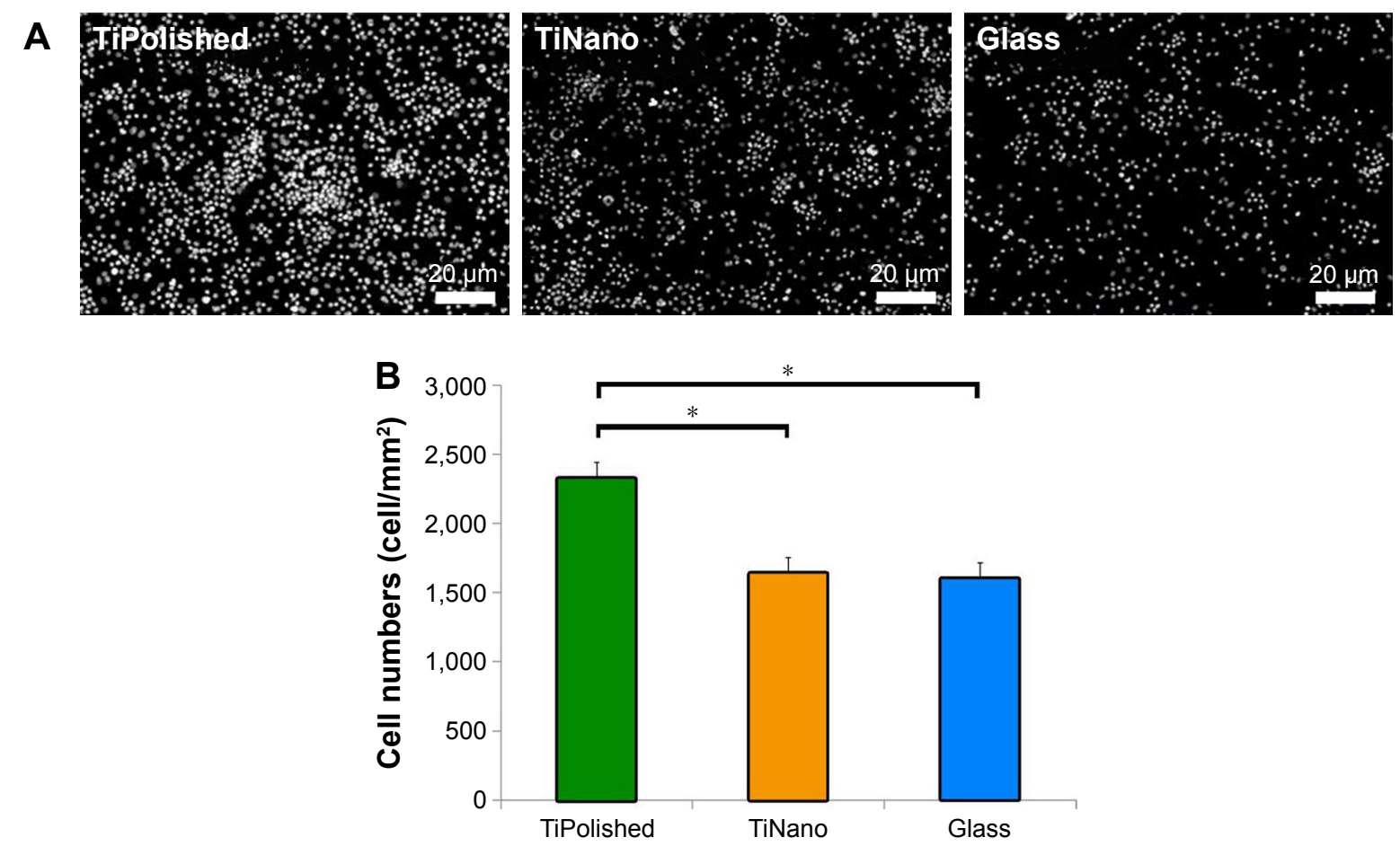

Figure 2 Visualization of cell density by staining of nuclei.

Notes: DAPI (A) and cell numbers (B) on TiPolished, TiNano, and Glass surfaces. $* P<0.05$; error bars, SD.

Abbreviations: TiNano, nanocavitated titanium; TiPolished, polished titanium; Glass, glass coverslips.
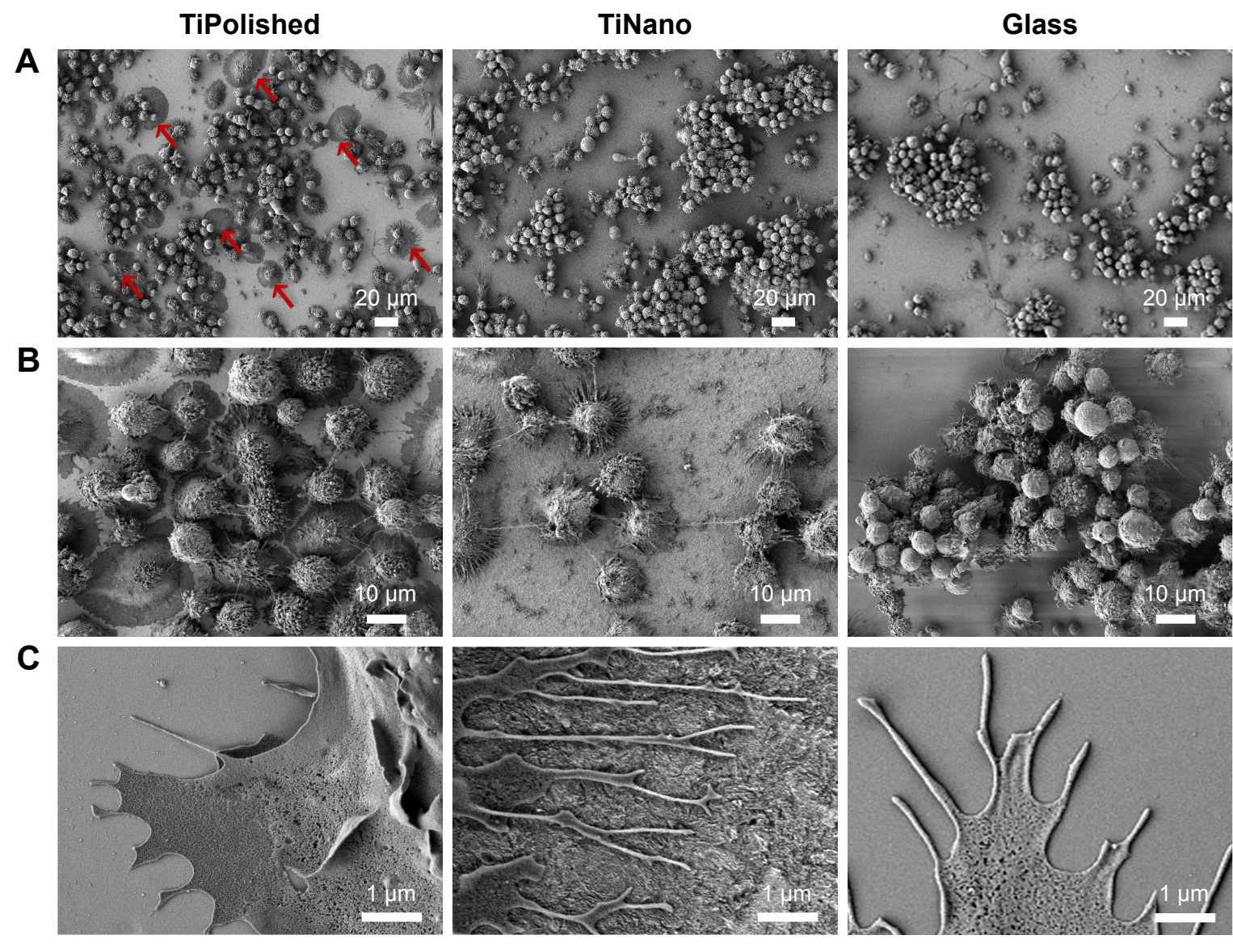

Figure 3 Field-emission scanning electron microscopy of U937 macrophages on TiPolished, TiNano, and Glass surfaces.

Notes: Macrophages spread less and adopted a more rounded profile on TiNano (A, B), filopodial extensions (C). Red arrows represent large cytoplasmic veils. Abbreviations: TiPolished, polished titanium; TiNano, nanocavitated titanium; Glass, glass coverslips. 


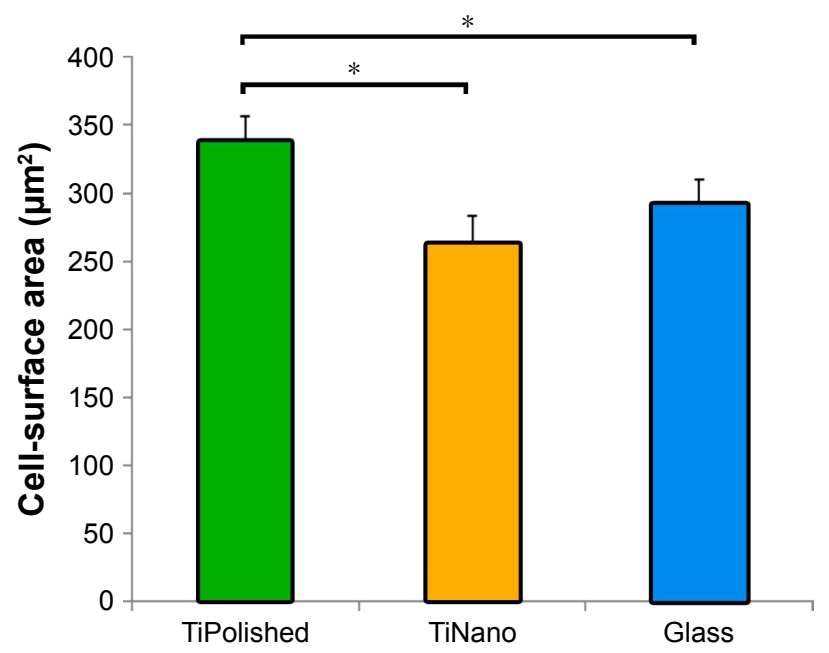

Figure 4 Cell-surface area on the three surfaces studied. Note: ${ }^{*} P<0.05$; error bars, $S D$.

Abbreviations: TiPolished, polished titanium; TiNano, nanocavitated titanium; Glass, glass coverslips.

labeling was seen on TiPolished and Glass (Figure 5A and B). This is consistent with the capacity of podosomes to sense and passively report topography. ${ }^{30}$ The podosomes on TiNano exhibited small actin dots in the center of the cell, suggesting that they represented inactive successor podosomes. ${ }^{29}$ These results showed that macrophages on TiPolished and to some extent on Glass were more active than the ones in contact with the TiNano surface.
Cell migration is broadly classified as either amoeboid or mesenchymal, and relies on multiple parameters, including morphological appearance..$^{31,32}$ Based on the latter, U937 cells on nanocavitated surfaces appeared to fit the amoeboid-migration mode classification, while those on TiPolished surfaces the mesenchymal one. This is further supported by the observation that there were fewer cells on TiNano. ${ }^{32} \mathrm{Lu}$ and Webster ${ }^{27}$ referred to this classification in a study on the response of IC21 macrophages to nano- and submicron-rough titanium. Although changes in structural features have been reported in macrophages cultured on various surfaces, the morphological classification conventionally used to describe them in inflammatory afflictions has not been systematically applied to categorize their behavior on nanotopographies. This classification could offer an additional parameter to better define the inflammatory response of macrophages to various biomaterials and surface topographies relating to their physiological role in inflammation. In addition, the use of a standard terminology may also facilitate comparisons among studies with various macrophage lineages and surfaces.

Different intracellular signaling pathways have been associated with the amoeboid and mesenchymal migration modes. The amoeboid-migration mode that typifies what we observed on TiNano surfaces is ROCK-dependent. ${ }^{28}$ This protein is also implicated in the response of osteogenic cells
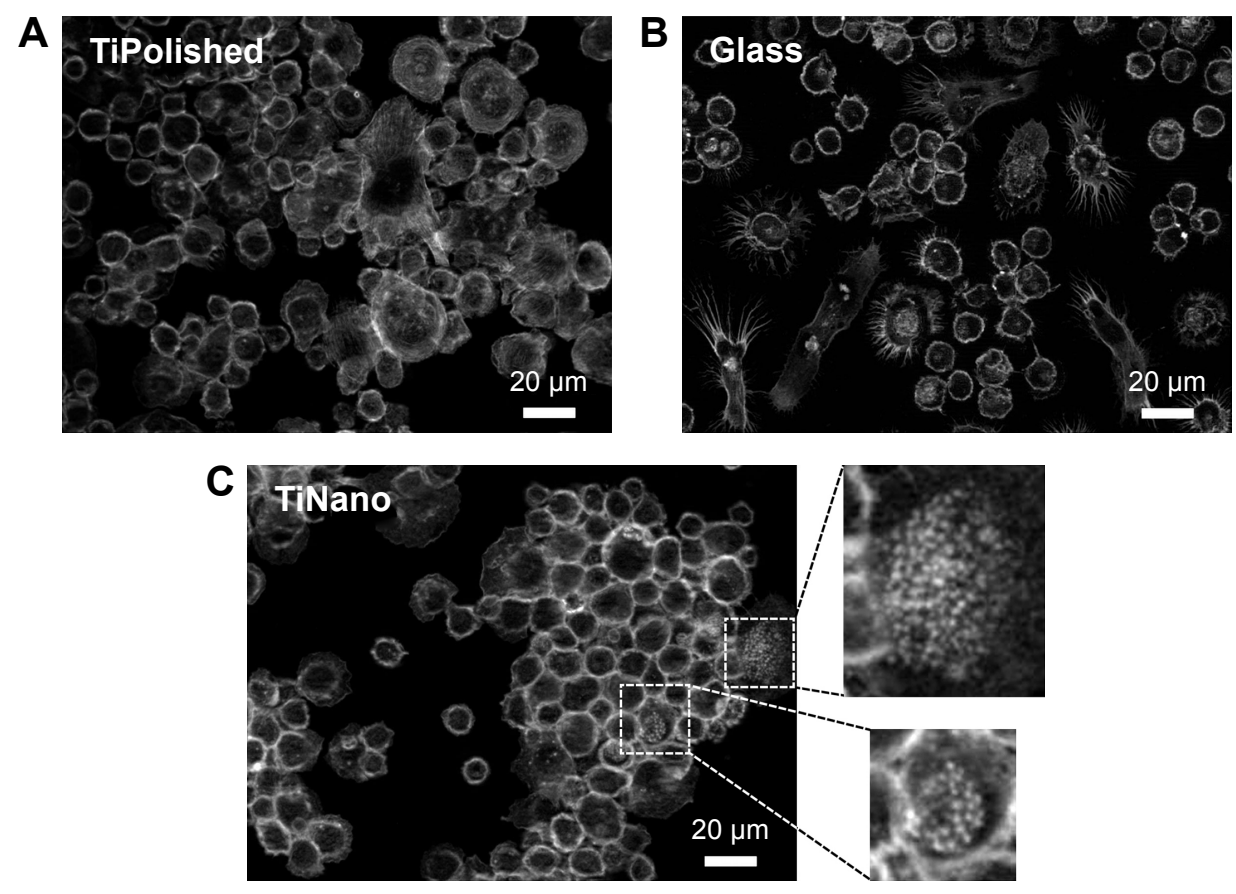

Figure 5 Actin labeling of macrophage on TiPolished (A), Glass (B), and TiNano (C).

Note: Enlargement of boxed areas shows podosome formation.

Abbreviations: TiPolished, polished titanium; Glass, glass coverslips; TiNano, nanocavitated titanium. 
cultured on mesoporous titanium surfaces. ${ }^{14}$ Indeed, similarly to U937 cells, osteogenic cells also undergo morphological changes and express abundant, thin, and long filopodia on both nanocavitated titanium and stainless steel. ${ }^{11,14} \mathrm{~A}$ recent work ${ }^{17}$ concluded that these structural changes were predominantly determined by the physical characteristics of surfaces, at least for MC3T3 osteogenic cells. The amoeboid morphology indicates greater macrophage mobility, and together with the lower number of cells, this may reflect a lesser attraction of U937 to TiNano.

\section{Macrophage expression of OPN, SPARC, and stabilin I}

OPN is an extracellular matrix protein and proinflammatory cytokine, and is expressed by macrophages and highly induced during inflammatory activation. ${ }^{33-35}$ While OPN was expressed on the three surfaces, in general it was less abundant on TiNano and Glass (Figure 6A). On a cellmorphology basis, the highest expression was found in elongated cells, which were more predominant on TiPolished.
Consequently, it can be concluded that the overall expression of OPN was greater on this surface.

SPARC and stabilin 1 are a protein and a receptor, respectively, which bind together and are expressed by macrophages in inflammatory conditions. ${ }^{36}$ SPARC is a secreted matricellular protein that promotes cellular deadhesion and motility in wound healing, carcinogenesis, and inflammation. ${ }^{36-38}$ Stabilin 1 is a scavenger receptor expressed on macrophages during chronic inflammation and tumorigenesis. ${ }^{39}$ To our knowledge, the in vitro expression of these two molecules by macrophages in relation to nanotopography has not been studied. High expression of SPARC and stabilin 1 was found in most cells on all three surfaces. Although there was a tendency for weaker labeling on TiNano (Figure 6B and C), the difference was too subtle to conclude unequivocally on a visual basis. This may be a case where rigorous quantitative analysis of the immunostaining and/ or reverse-transcription PCR analysis may provide a more accurate evaluation. The overall lower expression of OPN, and possibly also SPARC and stabilin 1, supports the concept
A

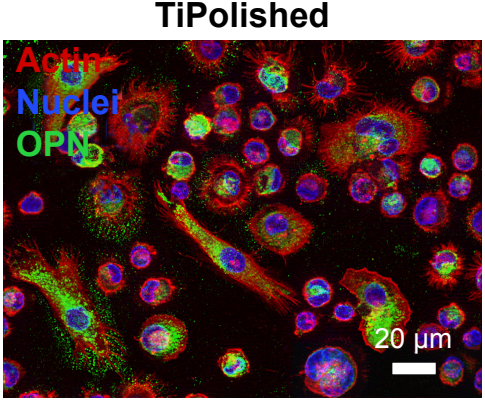

B

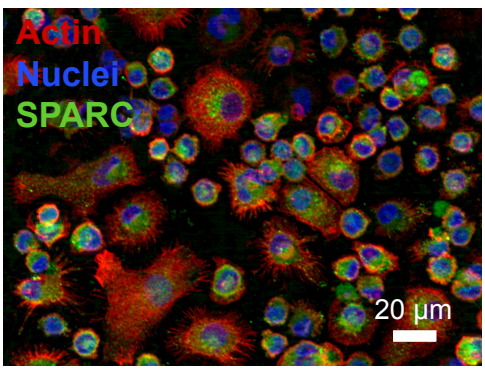

C

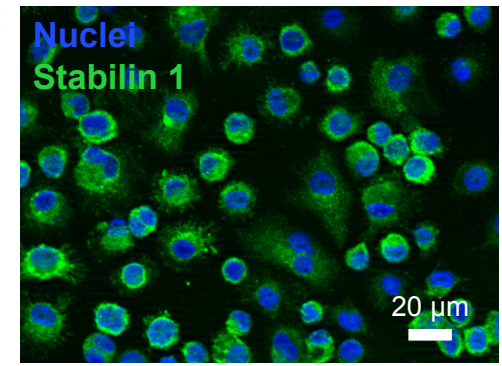

TiNano
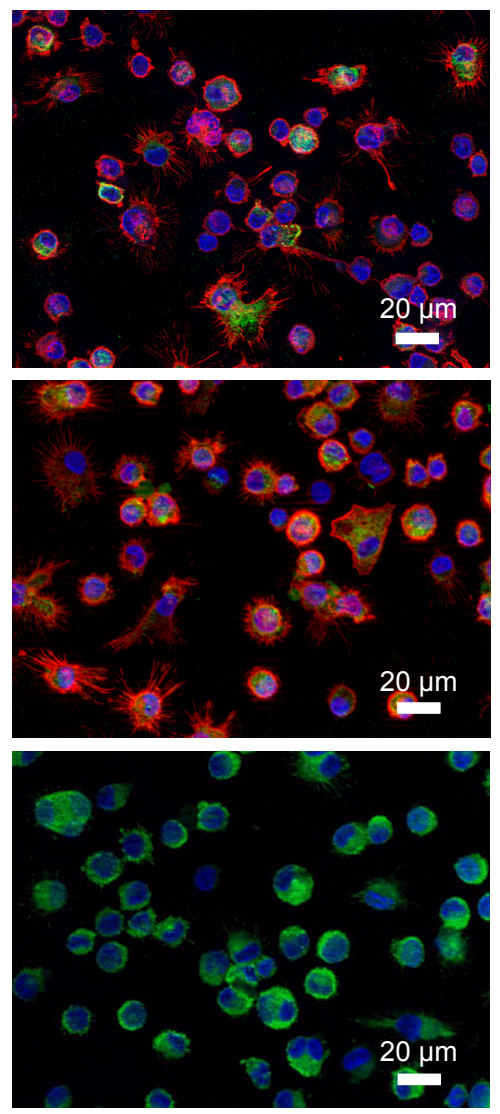

Glass
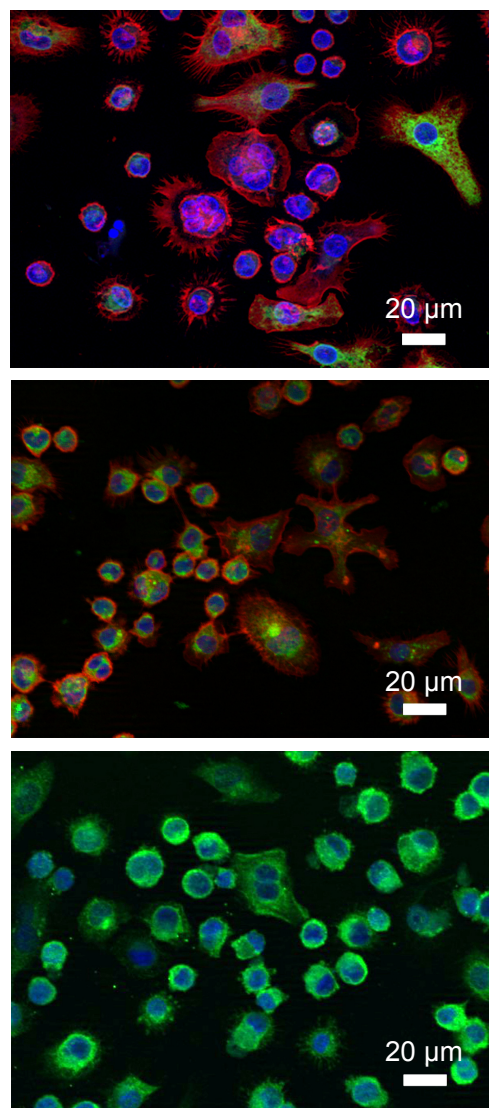

Figure 6 Macrophage protein expression.

Note: Osteopontin (A); SPARC (B); and stabilin I (C).

Abbreviations: TiPolished, polished titanium; TiNano, nanocavitated titanium; Glass, glass coverslips. 
that TiNano presents macrophages with more attenuated inflammatory cueing.

\section{Phagocytosis}

One of the functions of macrophages is the phagocytosis of cellular and acellular debris during inflammation and wound healing to allow the generation of new tissue. ${ }^{40,41}$ Cells on all three surfaces were capable of phagocytosing latex beads (Figure 7B), but there was a greater percentage of phagocytosing cells on TiNano compared to TiPolished (Figure 7A). Overall, there seemed to be more beads phagocytosed by the cells on TiNano and Glass surfaces (Figure 7B). Extrapolated in vivo, this heightened activity could forebode a better healing response.

\section{Influence of nanopatterning on cytokines}

The simple attachment and/or differentiation of macrophages onto a material surface does not allow judgment on whether those cells are more or less activated. The signals and enzymes that macrophages release, however, are indicative of their level of inflammatory activation. In this work, we have examined the influence of nanocavitated surfaces on stimulating the production of a wide number of cytokines (40), to gain insight into their functional activity and/or inflammatory potential. Of the multiple cytokines tested (Table 1), expression of the majority was similar on all three substrates, and some well-studied cytokines, such as CXCL13, CCL27, GM-CSF, and IL2, were released in very low concentrations (Table 1). I309/CCL1 showed a tendency to be upregulated on the nanostructured surface, but the difference was not statistically significant. MCP1/CCL2 was the only chemokine upregulated on TiNano (Figure 8). ENA78/CXCL5, eotaxin 3/CCL26, fractalkine/CX3CL1, GCP2/CXCL6, Groß/CXCL2, IP10/CXCL10, MIP3 $\alpha / C C L 20$, and MPIF1/CCL23 were all downregulated (Figure 8).

By focusing our analysis on those cytokines that showed strong differences among the three surfaces, some unique functional differences emerged. For example, there was a remarkable decrease in the amount of IP10/CXCL10 (78\%) and MIP3 $\alpha /$ CCL20 (67\%) expressed by cells on TiNano. These are all recognized as proinflammatory cytokines, and this observation confirms previous work on micro- and nanostructured materials. ${ }^{42,43}$ Our data also demonstrated $45 \%$ more release of the chemokine MCP1/CCL2 on TiNano, which is implicated in the recruitment of monocytes and macrophages to the implantation site. ${ }^{44}$ A perusal of the literature on nanoporous surfaces with similarity to ours indicated variable results

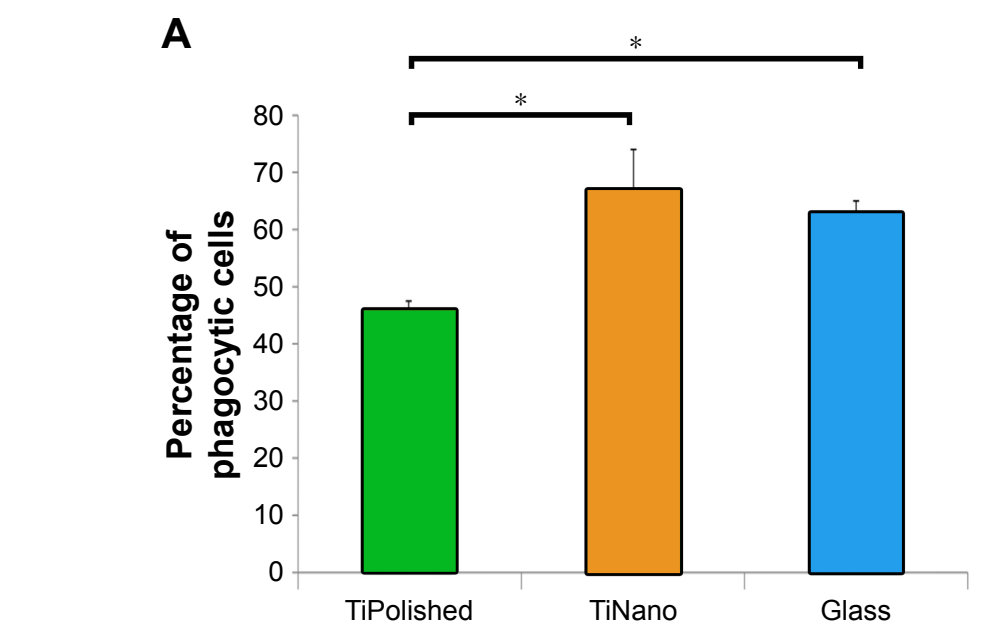

B
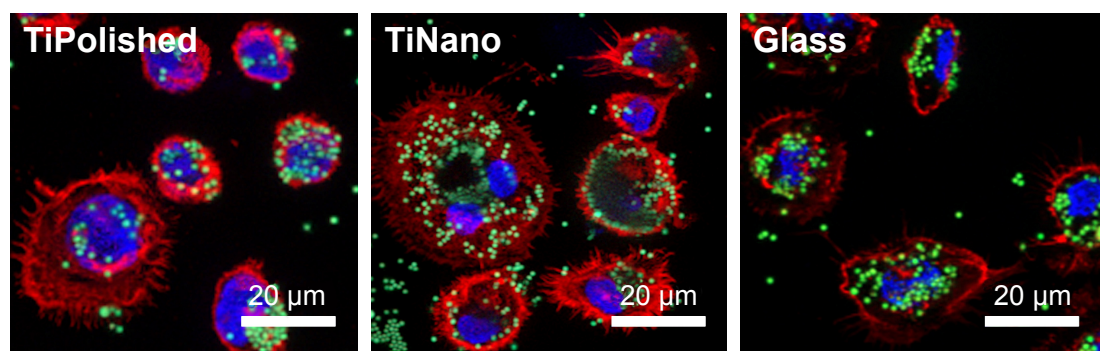

Figure 7 Phagocytosis of latex beads.

Notes: Counting of macrophage phagocytic activity $(\mathbf{A}) .{ }^{*} P<0.05$; error bars, SD. Representative fluorescence images of phagocytosis $(\mathbf{B})$ : actin (red), latex beads (green), and nuclei (blue).

Abbreviations: TiPolished, polished titanium; TiNano, nanocavitated titanium; Glass, glass coverslips. 
Table I Concentration of cytokine release from U937 macrophage cells on TiNano, TiPolished, and Glass

\begin{tabular}{|c|c|c|c|c|c|c|c|}
\hline \multirow[t]{2}{*}{ Cytokines } & \multirow[t]{2}{*}{ Gene } & \multicolumn{2}{|l|}{ TiPolished } & \multicolumn{2}{|l|}{ TiNano } & \multicolumn{2}{|l|}{ Glass } \\
\hline & & $\begin{array}{l}\text { Concentration } \\
(\mathrm{pg} / \mathrm{mL})\end{array}$ & SD & $\begin{array}{l}\text { Concentration } \\
(\mathrm{pg} / \mathrm{mL})\end{array}$ & SD & $\begin{array}{l}\text { Concentration } \\
(\mathrm{pg} / \mathrm{mL})\end{array}$ & SD \\
\hline ILI $\beta$ & $I L I B$ & 123.1 & 3.96 & 136.64 & 11.65 & 131.1 & 10.29 \\
\hline IL2 & IL2 & 8.34 & 0.76 & 7.68 & 0.87 & 8.68 & 1.03 \\
\hline IL4 & IL4 & 15.54 & 0.43 & 14.41 & 0.33 & 16.05 & 0.52 \\
\hline IL6 & IL6 & 19.97 & 0.89 & 22.08 & 2.74 & 20.86 & 0.92 \\
\hline IL8/CXCL8 & CXCL8 & $5,351.08$ & 423.02 & $5,126.36$ & 352.19 & $5,134.78$ & 129.80 \\
\hline ILIO & ILIO & 263.8 & 20.12 & 275.67 & 26.84 & 274.09 & 4.36 \\
\hline Pro-ILI6 & $1 L I 6$ & 114.95 & 3.71 & 101.82 & 3.93 & 117.21 & 9.42 \\
\hline GM-CSF & CSF2 & 17.06 & 0.99 & 12.97 & 0.54 & 14.54 & 0.27 \\
\hline $\mathrm{IFN} \gamma$ & IFNG & 48.82 & 1.01 & 40.55 & 1.63 & 47.74 & 0.99 \\
\hline MIF & MIF & $6,911.66$ & 933.97 & $6,198.58$ & 388.93 & $7,192.15$ & 946.83 \\
\hline TNF $\alpha$ & TNFA & 602.56 & 53.27 & 675.08 & 48.18 & 610.45 & 20.09 \\
\hline \multicolumn{8}{|l|}{ Chemokines } \\
\hline I309/CCLI & CCLI & $5,628.63$ & $2,186.93$ & $6,317.02$ & $1,325.03$ & $4,70 I .46$ & 250.33 \\
\hline $\mathrm{MCPI} / \mathrm{CCL} 2$ & CCL2 & $2,916.95$ & 163.07 & $4,255.06$ & I,035.87 & $2,973.05$ & 342.61 \\
\hline $\mathrm{MIPI} \alpha / C C L 3$ & CCL3 & 936.28 & 82.86 & 944.49 & 59.48 & 818.97 & 89.40 \\
\hline MCP3/CCL7 & CCL7 & $1,315.08$ & 50.80 & $1,268.9$ & 36.49 & I,235.84 & 38.50 \\
\hline MCP2/CCL8 & CCL8 & 995.02 & 237.14 & $1,3 \mid 2.55$ & 954.21 & $1,265.12$ & 780.12 \\
\hline Eotaxin/CCLII & CCLII & 22.54 & 0.70 & 20.25 & 0.33 & 22.53 & 0.30 \\
\hline MCP4/CCLI3 & $\mathrm{CCL} / 3$ & 14.94 & 14.94 & 12.33 & 12.33 & 15.18 & 0.48 \\
\hline MIPI $\delta / C C L I 5$ & CCLI5 & 13.65 & 1.12 & 7.15 & 0.23 & 16.48 & 1.69 \\
\hline TARC/CCLI7 & CCLI7 & 16.25 & 0.65 & 14 & 1.47 & 15.67 & 0.46 \\
\hline MIP3ß/CCLI9 & CCLI9 & 35.09 & 0.82 & 33.82 & 1.13 & 36.49 & 1.30 \\
\hline $\mathrm{MIP} 3 \alpha / \mathrm{CCL} 20$ & CCL2O & 596.63 & 88.52 & $195.4 \mid$ & 26.85 & 811.92 & 119.38 \\
\hline 6Ckine/CCL2I & CCL2I & 346.49 & 6.59 & 335.1 & 12.82 & 350.38 & 5.02 \\
\hline MDC/CCL22 & CCL22 & 13.91 & 1.41 & 15.21 & 0.33 & 14.6 & 0.35 \\
\hline MPIFI/CCL23 & CCL23 & 997.17 & 62.17 & 717.75 & 31.89 & $1,045.79$ & 28.54 \\
\hline Eotaxin 2/CCL24 & CCL24 & $3,591.95$ & 460.04 & $3,239.1$ & 467.83 & $3,858.11$ & 83.26 \\
\hline TECK/CCL25 & CCL25 & 796.23 & 7.47 & 717.82 & 19.38 & 780.23 & 13.07 \\
\hline Eotaxin 3/CCL26 & CCL26 & 145.55 & 8.70 & 60.92 & 2.03 & 194.4 & 16.83 \\
\hline CTACK/CCL27 & CCL27 & 5.67 & 0.22 & 4.87 & 0.30 & 5.38 & 0.23 \\
\hline Grol/CXCLI & CXCLI & 84.07 & 1.81 & 79.06 & 2.51 & 83.27 & 0.88 \\
\hline Groß/CXCL2 & CXCL2 & 241.69 & 26.29 & 66.96 & 4.54 & 327.16 & 36.67 \\
\hline ENA78/CXCL5 & CXCL5 & 965.66 & 48.33 & 782.48 & 15.96 & 885.28 & 54.47 \\
\hline GCP2/CXCL6 & CXCL6 & 105.57 & 11.39 & 40.18 & 1.97 & 139.39 & 13.12 \\
\hline MIG/CXCL9 & CXCL9 & 27.39 & 1.20 & 22.82 & 0.76 & 26.96 & 1.37 \\
\hline IPIO/CXCLIO & CXCLIO & 291.86 & 29.66 & 66.39 & 14.58 & 611.86 & 99.32 \\
\hline ITAC/CXCLII & CXCLIII & 27.05 & 3.06 & 6.85 & 1.26 & 44.49 & 7.52 \\
\hline SDFI/CXCLI 2 & CXCLI2 & 46.41 & 1.68 & 42.31 & 2.14 & 46.64 & 2.38 \\
\hline $\mathrm{BCAI} / \mathrm{CXCLI} 3$ & CXCLI3 & 3.77 & 0.17 & 3.18 & 0.18 & 3.71 & 0.09 \\
\hline SCYBI6/CXCLI6 & CXCLI6 & 356.22 & 18.02 & 388.62 & 12.05 & 381.25 & 25.65 \\
\hline Fractalkine/CX3CLI & CX3CLI & 686.63 & 41.17 & 318.29 & 36.37 & 833.76 & 101.37 \\
\hline
\end{tabular}

Abbreviations: TiPolished, polished titanium; TiNano, nanocavitated titanium; Glass, glass coverslips.

on the regulation of cytokine secretion. For example, Tan et $\mathrm{a}^{45}$ showed that the murine macrophage-like RAW264.7 cells on titanium nanotubes $\sim 100 \mathrm{~nm}$ in diameter were restricted in proliferation, migration, and proinflammatory cytokine expression (TNF $\alpha$, IL1 $\beta$, IL6). Ma et al ${ }^{7}$ compared different pore diameters of nanotubes created on titanium and 


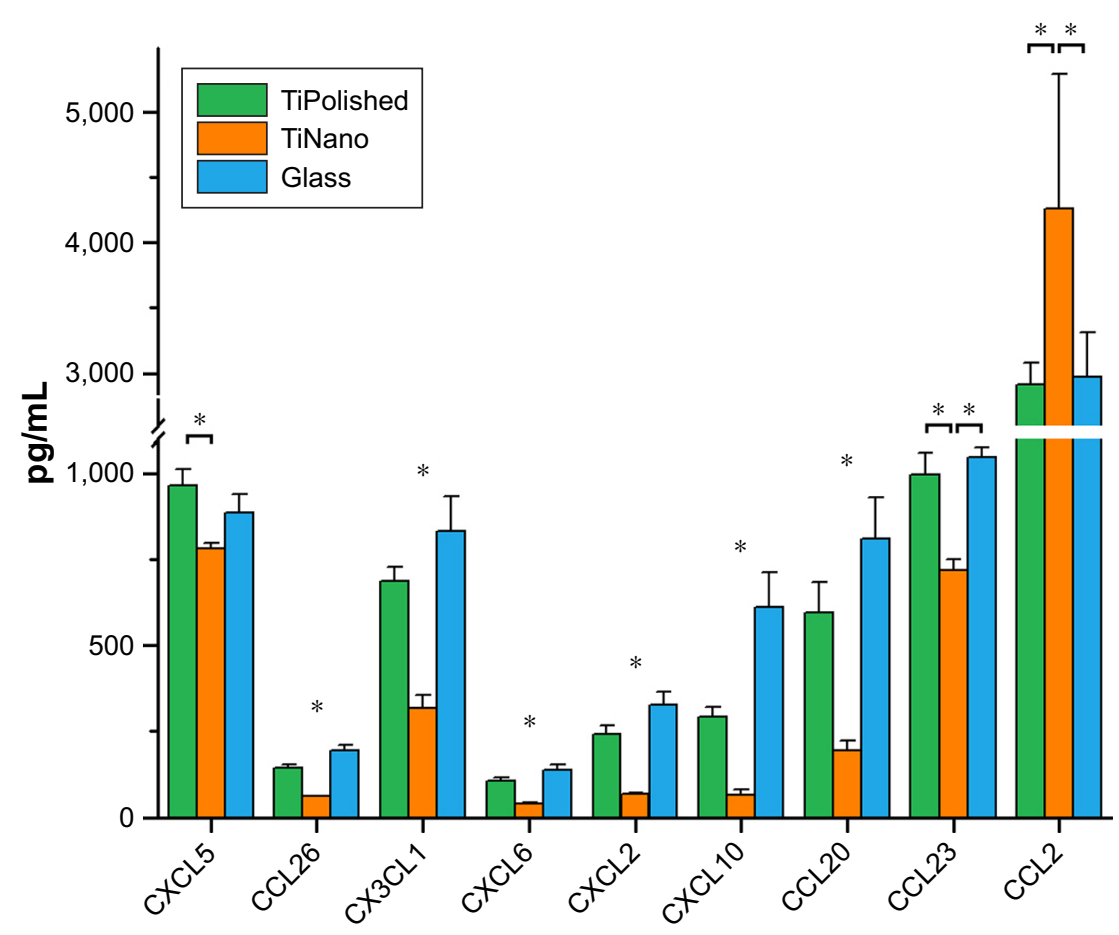

Figure 8 Influence of nanopatterning on cytokines: inflammatory cytokine release by macrophages on TiPolished, TiNano, and Glass surfaces. Note: $* P<0.05$; error bars, SD.

Abbreviations: TiPolished, polished titanium; TiNano, nanocavitated titanium; Glass, glass coverslips.

found a size effect on regulation of IL1 $\beta$, IL4, IL6, IL8, IL10, PDGFBB, TNF $\alpha$, and IFN $\gamma$. In contrast, Lu and Webster ${ }^{27}$ showed that expression of proinflammatory cytokines IL1 $\beta$, IL6, and TNF $\alpha$ by mouse IC21 macrophage cells was undetectable on both nanotopographic and flat surfaces without the presence of an inflammatory activator. These discrepancies likely relate to differences in the surfaces and macrophage cell lines used and the subset of cytokines examined, which complicates comparison between studies.

Taken together, our results indicate that nanocavitated surfaces exhibit a less inflammatory profile. Because such surfaces have been shown to promote osteogenic cell activity ${ }^{11,12,23}$ in vitro and bone formation in both $\operatorname{dog}^{23}$ and rat models,${ }^{15}$ it could be presumed that the macrophagic response they elicit do not interfere with bone formation and may actually facilitate the initial events leading to bone integration. Coculturing of osteogenic and macrophagic cells on mesoporous titanium surfaces could allow dissection of any bone-signaling pathways to which macrophages contribute.

\section{Conclusion}

A key aspect in the design of biomedical implants is the ability to minimize the inflammatory response and foreign-body reactions. We tested a distinctive mesoporous titanium surface obtained by a process of a simple chemical nanocavitation ${ }^{11}$ that selectively influences cell activity, ${ }^{12,23}$ but that has not yet been tested for its anti-inflammatory potential. Our results indicate that its physicochemical properties are sensed by U937 macrophagic cells. Collectively, the amoeboid morphology, phagocytosis activity, and cytokine profile elicited suggest that the generated surface trends toward a low inflammatory profile that could facilitate the integration of medically relevant metals in the body.

\section{Acknowledgments}

MBA was a recipient of a postdoctoral fellowship from the Fonds de Recherche du Québec - Santé. AN holds the Canada Research Chair in Calcified Tissues, Biomaterials, and Structure Imaging. The study was supported by the Canadian Institutes of Health Research (CIHR), Natural Sciences and Engineering Research Council of Canada (NSERC, RGPIN2016-04764), Canada Foundation for Innovation (CFI-32180), and the Canada Research Chair program. GI's current address is Departments of Biomedical and Odontostomatological Sciences and Morphological and Functional Images, School of Dentistry, University of Messina, Messina, Italy.

\section{Author contributions}

All authors made substantial contributions to conception and design, acquisition of data, or analysis and interpretation of data; took part in drafting the article or revising it critically for important intellectual content; gave final approval of the 
version to be published; and agree to be accountable for all aspects of the work.

\section{Disclosure}

The authors report no conflicts of interest in this work.

\section{References}

1. Anderson JM, Rodriguez A, Chang DT. Foreign body reaction to biomaterials. Semin Immunol. 2008;20(2):86-100.

2. Sheikh Z, Brooks PJ, Barzilay O, Fine N, Glogauer M. Macrophages, foreign body giant cells and their response to implantable biomaterials. Materials (Basel). 2015;8(9):5671-5701.

3. Neacsu P, Mazare A, Schmuki P, Cimpean A. Attenuation of the macrophage inflammatory activity by $\mathrm{TiO}_{2}$ nanotubes via inhibition of MAPK and NF-кB pathways. Int J Nanomedicine. 2015;10:6455-6467.

4. Wheeldon I, Farhadi A, Bick AG, Jabbari E, Khademhosseini A. Nanoscale tissue engineering: spatial control over cell-materials interactions. Nanotechnology. 2011;22(21):212001.

5. Chamberlain LM, Brammer KS, Johnston GW, Chien S, Jin S. Macrophage inflammatory response to $\mathrm{TiO} 2$ nanotube surfaces. J Biomater Nanobiotechnol. 2011;2:293-300.

6. Mohiuddin M, Pan HA, Hung YC, Huang GS. Control of growth and inflammatory response of macrophages and foam cells with nanotopography. Nanoscale Res Lett. 2012;7(1):394.

7. Ma QL, Zhao LZ, Liu RR, et al. Improved implant osseointegration of a nanostructured titanium surface via mediation of macrophage polarization. Biomaterials. 2014;35(37):9853-9867.

8. Neacsu P, Mazare A, Cimpean A, et al. Reduced inflammatory activity of RAW 264.7 macrophages on titania nanotube modified Ti surface. Int J Biochem Cell Biol. 2014;55:187-195.

9. Vetrone F, Variola F, Tambasco de Oliveira P, et al. Nanoscale oxidative patterning of metallic surfaces to modulate cell activity and fate. Nano Lett. 2009;9(2):659-665.

10. Yi JH, Bernard C, Variola F, et al. Characterization of a bioactive nanotextured surface created by controlled chemical oxidation of titanium. Surf Sci. 2006;600(19):4613-4621.

11. Rodriguez-Contreras A, Guadarrama Bello D, Flynn S, Variola F, Wuest JD, Nanci A. Chemical nanocavitation of surfaces to enhance the utility of stainless steel as a medical material. Colloids Surf B Biointerfaces. 2018;161:677-687.

12. de Oliveira PT, Zalzal SF, Beloti MM, Rosa AL, Nanci A. Enhancement of in vitro osteogenesis on titanium by chemically produced nanotopography. J Biomed Mater Res A. 2007;80(3):554-564.

13. Richert L, Vetrone F, Yi J-H, et al. Surface nanopatterning to control cell growth. Adv Mater. 2008;20:1488-1492.

14. Guadarrama Bello D, Fouillen A, Badia A, Nanci A. A nanoporous titanium surface promotes the maturation of focal adhesions and formation of filopodia with distinctive nanoscale protrusions by osteogenic cells. Acta Biomater. 2017;60:339-349.

15. Wazen RM, Kuroda S, Nishio C, Sellin K, Brunski JB, Nanci A. Gene expression profiling and histomorphometric analyses of the early bone healing response around nanotextured implants. Nanomedicine (Lond). 2013; 8(9):1385-1395.

16. Variola F, Zalzal SF, Leduc A, Barbeau J, Nanci A. Oxidative nanopatterning of titanium generates mesoporous surfaces with antimicrobial properties. Int J Nanomedicine. 2014;9:2319-2325.

17. Rodriguez-Contreras A, Guadarrama Bello D, Nanci A. Surface nanoporosity has a greater influence on osteogenic and bacterial cell adhesion than crystallinity and wettability. Appl Surf Sci. 2018;445:255-261.

18. Nanci A, Wuest JD, Péru L, et al. Chemical modification of titanium surfaces for covalent attachment of biological molecules. J Biomed Mater Res. 1998;40(2):324-335.

19. Matheson LA, Labow RS, Santerre JP. Biodegradation of polycarbonatebased polyurethanes by the human monocytes-derived macrophage and U937 cell systems. J Biomed Mater Res. 2002;61(4):505-513.
20. McDade JK, Brennan-Pierce EP, Ariganello MB, Labow RS, Michael Lee J. Interactions of U937 macrophage-like cells with decellularized pericardial matrix materials: influence of crosslinking treatment. Acta Biomater. 2013;9(7):7191-7199.

21. Fong D, Ariganello MB, Girard-Lauzière J, Hoemann CD. Biodegradable chitosan microparticles induce delayed STAT-1 activation and lead to distinct cytokine responses in differentially polarized human macrophages in vitro. Acta Biomater. 2015;12:183-194.

22. Lee HS, Stachelek SJ, Tomczyk N, Finley MJ, Composto RJ, Eckmann DM. Correlating macrophage morphology and cytokine production resulting from biomaterial contact. J Biomed Mater Res A. 2013; 101(1):203-212.

23. Tavares MG, de Oliveira PT, Nanci A, Hawthorne AC, Rosa AL, Xavier SP. Treatment of a commercial, machined surface titanium implant with $\mathrm{H} 2 \mathrm{SO} 4 / \mathrm{H} 2 \mathrm{O} 2$ enhances contact osteogenesis. Clin Oral Implants Res. 2007;18(4):452-458.

24. Rosa AL, Kato RB, Castro Raucci LM, et al. Nanotopography drives stem cell fate toward osteoblast differentiation through $\alpha 1 \beta 1$ integrin signaling pathway. J Cell Biochem. 2014;115(3):540-548.

25. Variola F, Lauria A, Nanci A, Rosei F. Influence of treatment conditions on the chemical oxidative activity of $\mathrm{H} 2 \mathrm{SO} 4 / \mathrm{H} 2 \mathrm{O} 2$ mixtures for modulating the topography of titanium. Adv Eng Mater. 2009;11(12): B227-B234.

26. Hind LE, Dembo M, Hammer DA. Macrophage motility is driven by frontal-towing with a force magnitude dependent on substrate stiffness. Integr Biol (Camb). 2015;7(4):447-453.

27. Lu J, Webster TJ. Reduced immune cell responses on nano and submicron rough titanium. Acta Biomater. 2015;16:223-231.

28. Van Goethem E, Poincloux R, Gauffre F, Maridonneau-Parini I, Le Cabec V. Matrix architecture dictates three-dimensional migration modes of human macrophages: differential involvement of proteases and podosome-like structures. J Immunol. 2010;184(2):1049-1061.

29. Bhuwania R, Cornfine S, Fang Z, Krüger M, Luna EJ, Linder S. Supervillin couples myosin-dependent contractility to podosomes and enables their turnover. J Cell Sci. 2012;125(Pt 9):2300-2314.

30. Linder S, Wiesner C. Feel the force: Podosomes in mechanosensing. Exp Cell Res. 2016;343(1):67-72.

31. Paluch EK, Aspalter IM, Sixt M. Focal adhesion-independent cell migration. Annu Rev Cell Dev Biol. 2016;32:469-490.

32. O'Neill PR, Castillo-Badillo JA, Meshik X, Kalyanaraman V, Melgarejo K, Gautam N. Membrane flow drives an adhesionindependent amoeboid cell migration mode. Dev Cell. 2018;46(1): 9-22.e4.

33. Kahles F, Findeisen HM, Bruemmer D. Osteopontin: a novel regulator at the cross roads of inflammation, obesity and diabetes. Mol Metab. 2014; 3(4):384-393.

34. WolakT.Osteopontin-amulti-modalmarkerandmediatorinatherosclerotic vascular disease. Atherosclerosis. 2014;236(2):327-337.

35. Zhao C, Fancy SP, Ffrench-Constant C, Franklin RJ. Osteopontin is extensively expressed by macrophages following CNS demyelination but has a redundant role in remyelination. Neurobiol Dis. 2008;31(2): 209-217.

36. Workman G, Sage EH. Identification of a sequence in the matricellular protein SPARC that interacts with the scavenger receptor stabilin-1. J Cell Biochem. 2011;112(4):1003-1008.

37. Sangaletti S, Tripodo C, Cappetti B, et al. SPARC oppositely regulates inflammation and fibrosis in bleomycin-induced lung damage. Am J Pathol. 2011;179(6):3000-3010.

38. Nagaraju GP, Dontula R, El-Rayes BF, Lakka SS. Molecular mechanisms underlying the divergent roles of SPARC in human carcinogenesis. Carcinogenesis. 2014;35(5):967-973.

39. Kzhyshkowska J, Gratchev A, Goerdt S. Stabilin-1, a homeostatic scavenger receptor with multiple functions. J Cell Mol Med. 2006;10(3): 635-649.

40. Hotchkiss KM, Reddy GB, Hyzy SL, Schwartz Z, Boyan BD, OlivaresNavarrete R. Titanium surface characteristics, including topography and wettability, alter macrophage activation. Acta Biomater. 2016;31: $425-434$. 
41. Scull CM, Hays WD, Fischer TH. Macrophage pro-inflammatory cytokine secretion is enhanced following interaction with autologous platelets. J Inflamm (Lond). 2010;7:53.

42. Bartneck M, Schulte VA, Paul NE, Diez M, Lensen MC, ZwadloKlarwasser G. Induction of specific macrophage subtypes by defined micro-patterned structures. Acta Biomater. 2010;6(10):3864-3872.

43. Barth KA, Waterfield JD, Brunette DM. The effect of surface roughness on RAW 264.7 macrophage phenotype. J Biomed Mater Res A. 2013; 101(9):2679-2688.
44. Sato T, Pajarinen J, Behn A, et al. The effect of local IL-4 delivery or CCL2 blockade on implant fixation and bone structural properties in a mouse model of wear particle induced osteolysis. J Biomed Mater Res A. 2016;104(9):2255-2262.

45. Tan J, Zhao C, Wang Y, et al. Nano-topographic titanium modulates macrophage response in vitro and in an implant-associated rat infection model. RSC Adv. 2016;6(113):111919-111927.

\section{Publish your work in this journal}

The International Journal of Nanomedicine is an international, peerreviewed journal focusing on the application of nanotechnology in diagnostics, therapeutics, and drug delivery systems throughout the biomedical field. This journal is indexed on PubMed Central, MedLine, CAS, SciSearch ${ }^{\circledR}$, Current Contents ${ }^{\circledR} /$ Clinical Medicine,
Journal Citation Reports/Science Edition, EMBase, Scopus and the Elsevier Bibliographic databases. The manuscript management system is completely online and includes a very quick and fair peer-review system, which is all easy to use. Visit http://www.dovepress.com/ testimonials.php to read real quotes from published authors. 